\title{
On the Fourier dimension of $(d, k)$-sets and Kakeya sets with restricted directions
}

\author{
Jonathan M. Fraser ${ }^{1}$ - Terence L. J. Harris ${ }^{2}$ - Nicholas G. Kroon ${ }^{1}$
}

Received: 28 May 2021 / Accepted: 4 January 2022 / Published online: 24 February 2022

(c) The Author(s) 2022

\begin{abstract}
$\mathrm{A}(d, k)$-set is a subset of $\mathbb{R}^{d}$ containing a $k$-dimensional unit ball of all possible orientations. Using an approach of D. Oberlin we prove various Fourier dimension estimates for compact $(d, k)$-sets. Our main interest is in restricted $(d, k)$-sets, where the set only contains unit balls with a restricted set of possible orientations $\Gamma$. In this setting our estimates depend on the Hausdorff dimension of $\Gamma$ and can sometimes be improved if additional geometric properties of $\Gamma$ are assumed. We are led to consider cones and prove that the cone in $\mathbb{R}^{d+1}$ has Fourier dimension $d-1$, which may be of interest in its own right.
\end{abstract}

Keywords Fourier dimension · Kakeya set $\cdot(d, k)$-set $\cdot$ Hausdorff dimension

Mathematics Subject Classification Primary 42B10 $\cdot 28 \mathrm{~A} 80 \cdot 28 \mathrm{~A} 78$

\section{Kakeya sets, $(d, k)$-sets, and dimension theory}

A Kakeya set is a subset of $\mathbb{R}^{d}$ containing a unit line segment in every direction. Besicovitch [1] proved that there exist Kakeya sets with zero $d$-dimensional Lebesgue measure (for any $d \geq 2$ ) and it is a notorious problem in geometric measure theory and harmonic analysis to determine if Kakeya sets can be even smaller than this, that is, can they have Hausdorff dimension strictly less than $d$ ? The case $d=1$ is trivial and we assume throughout that $d \geq 2$. Davies proved that Kakeya sets in $\mathbb{R}^{2}$ must have Hausdorff dimension 2 [3]. The problem

JMF was financially supported by an EPSRC Standard Grant (EP/R015104/1) and a Leverhulme Trust Research Project Grant (RPG-2019-034).

Jonathan M. Fraser

jmf32@st-andrews.ac.uk

Terence L. J. Harris

tlh236@cornell.edu

Nicholas G. Kroon

nkroon3@gmail.com

1 School of Mathematics and Statistics, University of St Andrews, St. Andrews, Scotland

2 Department of Mathematics, Cornell University, Ithaca, NY 14853, USA 
is open for $d \geq 3$ but various partial results are known. Notably, it was proved in [11] that Kakeya sets in $\mathbb{R}^{3}$ have Hausdorff dimension at least $5 / 2+\varepsilon$ for some small constant $\varepsilon>0$ and, in the general case, it was proved in [10] that Kakeya sets have Hausdorff dimension at least $(2-\sqrt{2})(d-4)+3$. Further improvements in certain dimensions were achieved recently in [9]. See [9,fig. 5] for a survey of the state of the art.

Oberlin gave a Fourier analytic proof of Davies' result [17] which actually establishes something stronger: a compact Kakeya set in $\mathbb{R}^{2}$ must have Fourier dimension 2. Oberlin's result and proof are the starting point for our work and we use his general approach to study variants of the Kakeya problem, especially $(d, k)$-sets with restricted orientations.

A $(d, k)$-set is a subset of $\mathbb{R}^{d}$ containing a $k$-dimensional unit ball of all possible orientations. As such, $(d, 1)$-sets are Kakeya sets. We give a more formal definition below. One can now ask if $(d, k)$-sets with zero $d$-dimensional Lebesgue measure exist for $d>k \geq 2$ ? In fact, this is an open problem in general but it is conjectured that no such sets exist. Falconer [5] proved that $(d, k)$-sets have positive measure whenever $k>d / 2$. Around the same time Marstrand [13] proved that $(3,2)$-sets have positive 3-dimensional measure using a different approach. This result has subsequently been strengthened by Bourgain [2] and Oberlin [19]. As far as we know the state of the art is that $(d, k)$-sets necessarily have positive Lebesgue measure when $(1+\sqrt{2})^{k-1}+k>d$, see [15,Chapter 24] and the survey [16].

Our main interest is in restricted $(d, k)$-sets, which we introduce now, and in estimates for the Fourier dimension. The Grassmannian manifold $G(d, k)$ consists of all $k$-dimensional subspaces of $\mathbb{R}^{d}$. This is a smooth compact manifold of dimension $k(d-k)$, see [14]. To formally define $(d, k)$-sets it is convenient to associate each subspace $s \in G(d, k)$ with an orthonormal basis $\left\{x_{1}^{s}, x_{2}^{s}, \ldots, x_{k}^{s}\right\} \subseteq \mathbb{R}^{d}$. In what follows it should be clear that the specific choice of basis is irrelevant. Let $\Gamma \subseteq G(d, k)$. If $E \subseteq \mathbb{R}^{d}$ is a $(d, k, \Gamma)$-set, then for all $s \in \Gamma$ there exists a translation $t_{s} \in \mathbb{R}^{d}$ such that

$$
t_{s}+\sum_{i=1}^{k} r_{i} x_{i}^{s} \in E
$$

for all $r=\left(r_{1}, r_{2}, \ldots, r_{k}\right) \in[0,1]^{k}$. In particular, a $(d, k)$-set is a $(d, k, G(d, k))$-set. We aim to bound the Fourier dimension of compact $(d, k, \Gamma)$-sets in terms of $d, k$, and geometric properties of $\Gamma$. The Fourier dimension of a set is bounded above by the Hausdorff dimension, hence lower bounds for the Fourier dimension give lower bounds for Hausdorff dimension. A related problem was considered by Oberlin [18]. This paper considers sets in $\mathbb{R}^{d}$ containing certain families of affine hyperplanes. If the Hausdorff dimension of the family is large enough, then it is proved in [18, Theorem 1.3] that the Lebesgue measure of the set must be positive.

The Fourier transform of a Lebesgue integrable, complex-valued function $f$ on $\mathbb{R}^{d}$ is the function $\hat{f}: \mathbb{R}^{d} \rightarrow \mathbb{C}$ given by

$$
\hat{f}(\xi)=\int_{\mathbb{R}^{d}} f(x) e^{-2 \pi i \xi \cdot x} \mathrm{~d} x .
$$

Analogously, the Fourier transform of a Borel measure $\mu$ on $\mathbb{R}^{d}$ is the function $\hat{\mu}: \mathbb{R}^{d} \rightarrow \mathbb{C}$ given by

$$
\hat{\mu}(\xi)=\int_{\mathbb{R}^{d}} e^{-2 \pi i \xi \cdot x} \mathrm{~d} \mu(x) .
$$

We write $\mathcal{M}(E)$ to denote the set of all Borel probability measures supported on a closed set $E$. Throughout we write $A \lesssim B$ to mean there is a constant $c>0$ such that $A \leq c B$. 
If the implicit constant $c$ depends on another parameter $\varepsilon$ we write $A \lesssim_{\varepsilon} B$. The Fourier dimension of a closed set $E \subseteq \mathbb{R}^{d}$ is then

$$
\operatorname{dim}_{\mathrm{F}} E=\sup \left\{0 \leq s \leq d: \exists \mu \in \mathcal{M}(E) \text { such that }|\hat{\mu}(\xi)| \lesssim s|\xi|^{-s / 2}\right\} .
$$

By relating energy to the Fourier transform, it is straightforward to see that $\operatorname{dim}_{\mathrm{F}} E \leq \operatorname{dim}_{\mathrm{H}} E$, see [15], where $\operatorname{dim}_{H}$ denotes Hausdorff dimension.

There exist sets in $\mathbb{R}^{d}$ with Fourier dimension $d$ but zero $d$-dimensional Lebesgue measure. For example, Besicovitch sets in $\mathbb{R}^{2}$. However, if we know that the Fourier decay of a measure $\mu \in \mathcal{M}(E)$ is "even better" than is required for full dimension, i.e. if there exists $\varepsilon>0$ such that

$$
|\hat{\mu}(\xi)| \lesssim_{\varepsilon}|\xi|^{-(d+\varepsilon) / 2}
$$

then $E \subseteq \mathbb{R}^{d}$ not only has full Fourier dimension, but also positive $d$-dimensional Lebesgue measure. Interestingly, there is no implication in the other direction. Indeed, there exist compact subsets of $\mathbb{R}^{d}$ with positive Lebesgue measure but with Fourier dimension equal to 0 , see [4,Example 18]. In particular, none of the Fourier dimension estimates we provide in this paper are implied by statements about positivity of Lebesgue measure.

We metrise $G(d, k)$ using the Hausdorff distance. The Hausdorff distance between two non-empty compact subsets $A, B$ of a compact metric space $(X, d)$ is given by

$$
d_{H}(A, B)=\max \left\{\sup _{a \in A} \inf _{b \in B} d(a, b), \sup _{b \in B} \inf _{a \in A} d(b, a)\right\} .
$$

The metric we use on $G(d, k)$ is then

$$
d_{(d, k)}(s, t)=d_{H}\left(s \cap S^{d-1}, t \cap S^{d-1}\right)
$$

for $s, t \in G(d, k)$ and where $S^{d-1}$ is the unit sphere in $\mathbb{R}^{d}$.

\section{Results and applications}

We state our main result in terms of an abstract scaling condition, which we refer to as $\beta$ scaling. We then apply this result in two different ways to obtain less abstract corollaries. Given $\xi \in \mathbb{R}^{d} \backslash\{0\}$ and $\eta>0$, let

$$
S_{\xi, \eta}=\left\{s \in G(d, k):\left|\xi \cdot x_{i}^{s}\right|<\eta|\xi|, \forall i=1, \ldots, k\right\} .
$$

Essentially, $s \in S_{\xi, \eta}$ if it is in an $\eta$-neighbourhood of the orthogonal complement of $\xi$. We say $\Gamma \subseteq G(d, k)$ is $\beta$-scaling for $\beta \geq 0$ if it supports a Borel measure $\gamma$ such that

$$
\gamma\left(S_{\xi, \eta}\right) \lesssim_{\beta} \eta^{\beta}
$$

for all $\xi \in \mathbb{R}^{d} \backslash\{0\}$ and $\eta>0$.

Theorem 2.1 Let $\Gamma \subseteq G(d, k)$ and assume $\Gamma$ is $\beta$-scaling. Let $E \subseteq \mathbb{R}^{d}$ be a compact $(d, k, \Gamma)$-set. Then

$$
\operatorname{dim}_{\mathrm{F}} E \geq \min \{2 \beta, d\} .
$$

Moreover, if $\beta>d / 2$, then E has positive d-dimensional Lebesgue measure. 
We defer the proof of Theorem 2.1 to Sect. 3. Our first application of Theorem 2.1 is to bound the Fourier dimension of $(d, k, \Gamma)$-sets from below in terms of the Hausdorff dimension of $\Gamma$. The next result guarantees that $\Gamma$ is $\beta$-scaling if its Hausdorff dimension is sufficiently large.

Proposition 2.2 Let $\Gamma \subseteq G(d, k)$ and suppose $k(d-1-k)<b<\operatorname{dim}_{\mathrm{H}} \Gamma$. Then $\Gamma$ is $(b-k(d-1-k))$-scaling.

We defer the proof of Theorem 2.2 to Sect. 4. Combining Theorem 2.1 and Proposition 2.2 we immediately get the following result.

Corollary 2.3 Let $\Gamma \subseteq G(d, k)$ and $E \subseteq \mathbb{R}^{d}$ be a compact $(d, k, \Gamma)$-set. If $2\left(\operatorname{dim}_{\mathrm{H}} \Gamma-k(d-\right.$ $1-k)) \leq d$, then

$$
\operatorname{dim}_{\mathrm{F}} E \geq 2\left(\operatorname{dim}_{\mathrm{H}} \Gamma-k(d-1-k)\right) .
$$

Otherwise, if $2\left(\operatorname{dim}_{\mathrm{H}} \Gamma-k(d-1-k)\right)>d$, then $\operatorname{dim}_{\mathrm{F}} E=d$ and $E$ has positive $d$ dimensional Lebesgue measure.

The special case of Corollary 2.3 when $d=2>1=k$ was proved in [17]. The final conclusion of Corollary 2.3 giving conditions guaranteeing positive measure can be deduced from [18, Theorem 1.3], but our Fourier dimension estimates are new to the best of our knowledge. Corollary 2.3 is sharp in the sense that for arbitrary $d>k$ there exist $(d, k, \Gamma)$ sets $E$ with $\operatorname{dim}_{\mathrm{H}} \Gamma=k(d-1-k)$ and $\operatorname{dim}_{\mathrm{F}} E=0$. Such sets can be constructed by choosing $V \in G(d, d-1)$ and then $\Gamma$ to be all $k$-dimensional subspaces of $\mathbb{R}^{d}$ which lie in $V$. With this choice of $\Gamma$ in place, $V \cap B(0,1)$ is a compact $(d, k, \Gamma)$-set and has Fourier dimension 0 since any subset of a hyperplane always has Fourier dimension 0 .

Specialising to $(d, k)$-sets, we recover Falconer's result for $k>d / 2$ and obtain new Fourier dimension bounds.

Corollary 2.4 Let $E \subseteq \mathbb{R}^{d}$ be a compact $(d, k)$-set. If $k \leq d / 2$, then

$$
\operatorname{dim}_{\mathrm{F}} E \geq 2 k .
$$

Otherwise, if $k>d / 2$, then $\operatorname{dim}_{\mathrm{F}} E=d$ and $E$ has positive $d$-dimensional Lebesgue measure.

To motivate these results, briefly consider compact $(3,2)$-sets which are known to have positive 3-dimensional Lebesgue measure. Corollary 2.3 provides a strengthening of this result by giving the same conclusion with only the requirement that the set of orientations has Hausdorff dimension $>3 / 2$. Recall that the dimension of the set of available orientations is 2. However, a further improvement is possible using [18, Theorem 1.3]. From this result it may be shown that the conclusion of positive measure can be reached with a set of orientations of dimensions $>1$. Moreover, this is sharp since if $\operatorname{dim}_{H} \Gamma=1$, then $(3,2, \Gamma)$-sets can have zero 3-dimensional Lebesgue measure. For example, consider the product of a Besicovitch set $E^{\prime} \in \mathbb{R}^{2}$ (that is, a Kakeya set with zero 2-dimensional measure) with a unit line segment. By disintegration of 3-dimensional Lebesgue measure we see that $E=E^{\prime} \times[0,1]$ is a null set and, moreover, is a $(3,2, \Gamma)$ with $\operatorname{dim}_{H} \Gamma=1$. Higher dimensional versions of this construction are also possible but left to the reader.

Next we point out that Theorem 2.1 often gives an improvement over Corollary 2.3 if there is additional geometric information known about $\Gamma$. There are many examples possible here and so we just highlight some of our favourites. Generally, better estimates will be possible for sets $\Gamma$ which stay sufficiently far away from hyperplanes. 
Let $d \geq 3$ and identify $G(d, 1)$ and $S^{d-1}$ in the natural way. We say a set $\Gamma \subseteq G(d, 1)$ is a non-degenerate sphere if it is a $(d-2)$-dimensional sphere with diameter strictly less than 2 , that is, it is not the intersection of $S^{d-1}$ with a hyperplane.

Proposition 2.5 Let $\Gamma \subseteq G(d, 1)$ be a non-degenerate sphere. If $d=3$, then $\Gamma$ is $1 / 2$-scaling. If $d \geq 4$, then $\Gamma$ is 1 -scaling.

We defer the proof of Proposition 2.5 to Sect. 5. As a consequence, we get the following result in the setting of restricted Kakeya sets. This is a strict improvement over Corollary 2.3 which does not give any non-trivial lower bounds in this setting.

Corollary 2.6 Let $E \subseteq \mathbb{R}^{d}$ be a compact set containing a unit line segment in a non-degenerate sphere of directions. If $d=3$, then

$$
\operatorname{dim}_{\mathrm{F}} E \geq 1
$$

If $d \geq 4$, then

$$
\operatorname{dim}_{\mathrm{F}} E \geq 2 .
$$

Corollary 2.6 is sharp for $d=3,4$. This can be seen by following result about cones, which may be of interest in its own right.

Theorem 2.7 For $d \geq 1$, the cone

$$
\mathcal{C}^{d}=\left\{\left(\xi_{1}, \ldots, \xi_{d}, \xi_{d+1}\right) \in \mathbb{R}^{d+1}:\left|\left(\xi_{1}, \ldots, \xi_{d}\right)\right|=\left|\xi_{d+1}\right|\right\}
$$

in $\mathbb{R}^{d+1}$ has Fourier dimension $d-1$.

We defer the proof of Theorem 2.7 to Sect. 6 .

It is perhaps noteworthy that in the case $d \geq 4$ in the above, we get the same lower bound for the Fourier dimension as that for genuine Kakeya sets, where lines in every direction are present, not just in a non-degenerate sphere of directions, see Corollary 2.4. Moreover, the non-degeneracy condition is necessary in the above, since a hyperplane contains a unit line segment in a $(d-2)$-dimensional sphere of directions, but has Fourier dimension 0.

We note that Corollary 2.6 gives non-trivial lower bounds for the Hausdorff dimension of restricted Kakeya sets $E$ in the case $d \geq 4$. However, these bounds can be improved in all dimensions by noting that the orthogonal projection of such $E$ onto the hyperplane orthogonal to the subspace spanned by the centre of $\Gamma$ is a genuine Kakeya set living in ambient dimension $d-1$. Then, since Hausdorff dimension cannot increase under projection, one can bound $\operatorname{dim}_{\mathrm{H}} E$ from below by applying the state of the art estimates for the Kakeya problem. We observe that Fourier dimension can (rather easily) increase under projection, and so such a reduction is not possible for Fourier dimension.

\section{Proof of Theorem 2.1}

Write $C_{c}^{\infty}(\mathbb{R})$ for the space of infinitely differentiable functions with compact support. Let $\phi \in C_{c}^{\infty}(\mathbb{R})$ be non-negative with support spt $\phi \subseteq[0,1]$ and such that $\int_{0}^{1} \phi(x) \mathrm{d} x=1$. Observe that the Fourier transform of $\phi$ is bounded above by 1 since

$$
|\hat{\phi}(\xi)| \leq\left|\int_{0}^{1} \phi(x) e^{-2 \pi i \xi x} \mathrm{~d} x\right| \leq \int_{0}^{1} \phi(x)\left|e^{-2 \pi i \xi x}\right| \mathrm{d} x=1 .
$$


Moreover, $\phi$ is an element of the Schwartz space $\mathcal{S}(\mathbb{R})$, and so $|\hat{\phi}(\xi)|$ decays rapidly as $|\xi| \rightarrow \infty$, see [15,Chapter 3]. The following proof broadly follows Oberlin [17,Proposition 2], see also the exposition [15,Theorem 11.3]. Our main new idea is that, when considering balls in $\mathbb{R}^{d}$ instead of lines in $\mathbb{R}^{2}$, one needs more understanding of the geometry of $\Gamma$, which is achieved via $\beta$-scaling. Moreover, we use a natural extension of Oberlin's argument to higher dimensions which requires multiple uses of the Schwartz function $\phi$.

Proof (Proof of Theorem 2.1) Let $\gamma$ be the Borel measure supported on $E$ coming from the definition of $\beta$-scaling. Assume for now that the map $s \mapsto t_{s}$ coming from the definition of $E$ is measurable. We address the issue of measurability at the end of the proof using a standard discretisation approach. Since $s \mapsto t_{s}$ is measurable we can use the Riesz representation theorem, see [20,2.14 Theorem], to define a measure $\mu \in \mathcal{M}(E)$ by

$$
\int_{E} f \mathrm{~d} \mu=\int_{G(d, k)} \int_{[0,1]^{k}} f\left(t_{s}+\sum_{i=1}^{k} r_{i} x_{i}^{s}\right) \phi\left(r_{1}\right) \cdots \phi\left(r_{k}\right) \mathrm{d} r \mathrm{~d} \gamma(s)
$$

for continuous functions $f$ on $\mathbb{R}^{d}$.

Let $\xi \in \mathbb{R}^{d}$ with $|\xi|>1$. The Fourier transform of $\mu$ at $\xi \in \mathbb{R}^{d}$ is given by

$$
\hat{\mu}(\xi)=\int_{G(d, k)} \int_{[0,1]^{k}} e^{-2 \pi i\left(t_{s}+\sum_{i=1}^{k} r_{i} x_{i}^{s}\right) \cdot \xi} \phi\left(r_{1}\right) \cdots \phi\left(r_{k}\right) \mathrm{d} r \mathrm{~d} \gamma(s) .
$$

First we integrate out the translations by

$$
\int_{[0,1]^{k}} e^{-2 \pi i\left(t_{s}+\sum_{i=1}^{k} r_{i} x_{i}^{s}\right) \cdot \xi} \phi\left(r_{1}\right) \cdots \phi\left(r_{k}\right) \mathrm{d} r=e^{-2 \pi i t_{s} \cdot \xi} \prod_{i=1}^{k} \int_{0}^{1} e^{-2 \pi i r_{i} x_{i}^{s} \cdot \xi} \phi\left(r_{i}\right) \mathrm{d} r_{i} .
$$

Therefore

$$
\left|e^{-2 \pi i t_{s} \cdot \xi} \prod_{i=1}^{k} \int_{0}^{1} e^{-2 \pi i r_{i} x_{i}^{s} \cdot \xi} \phi\left(r_{i}\right) \mathrm{d} r_{i}\right|=\prod_{i=1}^{k}\left|\hat{\phi}\left(\xi \cdot x_{i}^{s}\right)\right|,
$$

and

$$
|\hat{\mu}(\xi)| \leq \int_{G(d, k)} \prod_{i=1}^{k}\left|\hat{\phi}\left(\xi \cdot x_{i}^{s}\right)\right| \mathrm{d} \gamma(s)
$$

We split the above integral into two pieces which are then bounded separately. Let $\eta>0$ and recall the sets

$$
S_{\xi, \eta}=\left\{s \in G(d, k):\left|\xi \cdot x_{i}^{s}\right|<\eta|\xi|, \forall i=1, \ldots, k\right\}
$$

used to define $\beta$-scaling. Notice that if $s \notin S_{\xi, \eta}$ then there is some $j \in\{1, \ldots, k\}$ such that $\left|\xi \cdot x_{j}^{s}\right| \geq \eta|\xi|$. We then use the fact that $\hat{\phi}$ is bounded above by 1 and rapidly decreasing to conclude that, for any $N>1$, there exists a constant $C_{N}$ such that

$$
\left|\hat{\phi}\left(\xi \cdot x_{j}^{s}\right)\right| \leq \frac{C_{N}}{\left|\xi \cdot x_{j}^{s}\right|^{N}} .
$$


We then have

$$
\begin{aligned}
\int_{G(d, k) \backslash S_{\xi, \eta}} \prod_{i=1}^{k}\left|\hat{\phi}\left(\xi \cdot x_{i}^{s}\right)\right| \mathrm{d} \gamma(s) & \leq \int_{G(d, k) \backslash S_{\xi, \eta}}\left|\hat{\phi}\left(\xi \cdot x_{j}^{s}\right)\right| \mathrm{d} \gamma(s) \\
& \leq \int_{G(d, k) \backslash S_{\xi, \eta}} \frac{C_{N}}{\left|\xi \cdot x_{j}^{s}\right|^{N}} \mathrm{~d} \gamma(s) \\
& \lesssim_{N}(\eta|\xi|)^{-N} .
\end{aligned}
$$

On the other hand, we have the simple estimate

$$
\int_{S_{\xi, \eta}} \prod_{i=1}^{k}\left|\hat{\phi}\left(\xi \cdot x_{i}^{s}\right)\right| \mathrm{d} \gamma(s) \leq \int_{S_{\xi, \eta}} \mathrm{d} \gamma(s)=\gamma\left(S_{\xi, \eta}\right) \lesssim \eta^{\beta}
$$

where the final inequality is the only place where we use the $\beta$-scaling property. This gives

$$
|\hat{\mu}(\xi)| \leq \int_{G(d, k)} \prod_{i=1}^{k}\left|\hat{\phi}\left(\xi \cdot x_{i}^{s}\right)\right| \mathrm{d} \gamma(s) \lesssim_{N} \eta^{\beta}+(\eta|\xi|)^{-N} .
$$

Now let $0<\alpha<1$ and set $\eta=|\xi|^{-\alpha}$. Then, setting $N=\frac{\alpha \beta}{1-\alpha}$ yields

$$
|\hat{\mu}(\xi)| \lesssim \alpha|\xi|^{-\alpha \beta}
$$

This proves $\operatorname{dim}_{\mathrm{F}} E \geq \min \{2 \alpha \beta, d\}$ and letting $\alpha \rightarrow 1$ gives $\operatorname{dim}_{\mathrm{F}} E \geq \min \{2 \beta, d\}$ as required. Moreover, if $\beta>d / 2, E$ has positive $d$-dimensional Lebesgue measure.

It remains to address the measurability issue mentioned earlier. If $s \mapsto t_{s}$ is not measurable then we discretise $\mu$ as follows. Let $\left\{z_{1}, z_{2}, \ldots, z_{m}\right\}$ be a maximal $(1 / n)$-separated set of points in $\Gamma$ and define a measure $\gamma_{n}$ by

$$
\gamma_{n}=C_{\gamma, n} \sum_{i=1}^{m} \gamma\left(B\left(z_{i}, 1 / n\right)\right) \delta_{z_{i}}
$$

where $C_{\gamma, n}$ is a normalisation constant chosen such that $\gamma_{n}(G(d, k))=\gamma(G(d, k))$ and $\delta_{z_{i}}$ is a Dirac mass at $z_{i}$. Then we may define measures $\mu_{n}$ by replacing $\gamma$ with $\gamma_{n}$ in (3.1). The argument given above shows that $\left|\hat{\mu}_{n}(\xi)\right| \lesssim_{\alpha}|\xi|^{-\alpha \beta}$ for $n$ sufficiently large. Moreover, $\left(\mu_{n}\right)_{n}$ converges weakly to a measure $\mu \in \mathcal{M}(E)$, and the proof above goes through with this measure.

\section{Proof of Proposition 2.2}

For $0<b<\operatorname{dim}_{\mathrm{H}} \Gamma$, Frostman's lemma, see [14,8.17. Theorem], guarantees the existence of a compactly supported measure $\gamma \in \mathcal{M}(\Gamma)$ such that,

$$
\gamma(B(s, r)) \lesssim_{b} r^{b}
$$

for all $s \in G(d, k)$ and $r>0$. Straight from the definition of $S_{\xi, \eta}$, there exists a constant $C \geq 1$ such that $S_{\xi, \eta} \subseteq T_{\xi, \eta}$ where

$$
T_{\xi, \eta}:=\left\{s \in G(d, k): d_{(d, k)}(s, t)<C \eta \text { for some } t \in G(d, k) \text { contained in } \xi^{\perp}\right\}
$$

where $d_{(d, k)}$ is the metric defined in (1.1). 
Let $G_{\xi}$ denote the set of $k$-dimensional subspaces of $\xi^{\perp}$ and note that $G_{\xi}$ is a Grassmannian manifold isomorphic to $G(d-1, k)$. From the definition of the metric $d_{(d, k)}$, we have for $t, t^{\prime} \in G_{\xi}$ that $d_{(d, k)}\left(t, t^{\prime}\right)=d_{(d-1, k)}\left(t, t^{\prime}\right)$, i.e. it is safe to use the metric $d_{(d, k)}$ on $G_{\xi}$.

Let

$$
\left\{B\left(t_{i}, \eta\right) \cap G_{\xi}: t_{i} \in G_{\xi}, 1 \leq i \leq N_{\eta}\right\}
$$

be a minimal covering of $G_{\xi}$ by $\eta$-balls noting that

$$
N_{\eta} \lesssim \eta^{-k(d-1-k)}
$$

where the exponent $k(d-1-k)$ comes from the dimension of $G_{\xi}$. For $s \in T_{\xi, \eta}$ there exists $t \in G_{\xi}$ with $d_{(d, k)}(s, t)<C \eta$ and $t_{i}$, the centre of a ball in the covering, such that $d_{(d, k)}\left(t, t_{i}\right)<\eta$. Therefore

$$
T_{\xi, \eta} \subseteq \bigcup_{i=1}^{N_{\eta}} B\left(t_{i},(C+1) \eta\right)
$$

allowing us to relate coverings of $G_{\xi}$ to coverings of $T_{\xi, \eta}$. Using (4.1), (4.2) and (4.3) we get

$$
\gamma\left(S_{\xi, \eta}\right) \leq \gamma\left(T_{\xi, \eta}\right) \leq \sum_{i=1}^{N_{\eta}} \gamma\left(B\left(t_{i},(C+1) \eta\right)\right) \lesssim_{b} \eta^{-k(d-1-k)} \eta^{b}=\eta^{b-k(d-1-k)}
$$

completing the proof.

\section{Proof of Proposition 2.5}

Let $\gamma$ be the normalised spherical measure on $\Gamma$. Let $\xi \in \mathbb{R}^{d} \backslash\{0\}$ and $\eta>0$. We may assume that $\eta$ is much smaller than the diameter of $\Gamma$. Estimating $\gamma\left(S_{\xi, \eta}\right)$ immediately reduces to estimating the $\gamma$-volume of the intersection of the sphere $\Gamma$ with the $\eta$-neighbourhood of a plane of the same dimension. This in turn reduces to understanding the intersection of spheres and planes. There are two types of such intersection: tangential and non-tangential. We need a parameter $r$ to make this distinction more quantitative. In the non-tangential case, the intersection of a $(d-2)$-dimensional sphere and a plane of the same dimension is itself a sphere of dimension $(d-3)$. (In the case $d=3$ we think of a 0 -dimensional sphere as two distinct points with centre given by the midpoint.) Let $r>0$ be the minimal distance from the centre of the intersection to the original sphere $\Gamma$. The case $r=0$ is then the tangential case. If $r \leq 3 \eta$, then $S_{\xi, \eta}$ is contained in a ball of radius $\lesssim \sqrt{\eta}$, where the square root comes from the basic geometry of a sphere. Therefore

$$
\gamma\left(S_{\xi, \eta}\right) \lesssim \sqrt{\eta}^{(d-2)}=\eta^{d / 2-1} .
$$

On the other hand, if $r>3 \eta$, then $S_{\xi, \eta}$ is contained in an $\eta / \sqrt{r}$-thickening of a sphere of dimension $(d-3)$ and diameter $\lesssim \sqrt{r}$. This can be covered by

$$
\lesssim\left(\frac{\sqrt{r}}{\eta}\right)^{d-3} \frac{\eta / \sqrt{r}}{\eta}=\eta^{3-d} r^{d / 2-2}
$$

many $\eta$-balls each of $\gamma$ measure $\lesssim \eta^{d-2}$. Therefore,

$$
\gamma\left(S_{\xi, \eta}\right) \lesssim \eta r^{d / 2-2} \lesssim \eta^{\min \{1, d / 2-1\}} .
$$

Therefore, $\Gamma$ is $\min \{1, d / 2-1\}$-scaling, proving the claim. 


\section{Proof of Theorem 2.7}

The case $d=1$ is trivial, so assume that $d \geq 2$. The lower bound $\operatorname{dim}_{\mathrm{F}} \mathcal{C}^{d} \geq d-1$ follows (for example) by using (6.5) below and considering the measure defined by

$$
f \mapsto \int_{\mathbb{R}} \psi(r) \int_{S^{d-1}} f(r x, r) d \sigma(x) d r,
$$

for any non-negative Borel function $f$, where $\sigma$ is the rotation invariant Borel probability measure on $S^{d-1}$, and $\psi$ is a bump function on [1,2] with $\int \psi=1$.

Suppose for a contradiction that $\operatorname{dim}_{\mathrm{F}} \mathcal{C}^{d}>d-1$. Then there exists $\alpha>d-1$ and a Borel probability measure $\mu$ on $\mathcal{C}^{d}$, such that

$$
|\widehat{\mu}(\xi)| \lesssim|\xi|^{-\alpha / 2} \quad \forall \xi \in \mathbb{R}^{d+1} .
$$

By symmetry, and by replacing $\mu$ with $f \mu$ for an appropriate bump function $f$ (see [4,Lemma 1]), it may be assumed that for some $\varepsilon>0$,

$$
\operatorname{spt} \mu \subseteq\left\{(\xi,|\xi|) \in \mathbb{R}^{d} \times \mathbb{R}: \varepsilon \leq|\xi| \leq 1 / \varepsilon\right\} .
$$

Let $v$ be the Borel probability measure on $\mathcal{C}^{d}$ defined by

$$
\int f d v=\int_{\mathbb{R}^{d} \times \mathbb{R}} \int_{S^{d-1}} f(|x| w, z) d \sigma(w) d \mu(x, z),
$$

for any non-negative Borel function $f$. Then

$$
\begin{aligned}
\widehat{v}(\xi) & =\int_{\mathbb{R}^{d} \times \mathbb{R}} \int_{S^{d-1}} e^{-2 \pi i\langle\xi,(|x| w, z)\rangle} d \sigma(w) d \mu(x, z) \\
& =\int_{\mathbb{R}^{d} \times \mathbb{R}} \int_{O(d)} e^{-2 \pi i\langle\xi,(U x, z)\rangle} d \lambda(U) d \mu(x, z) \\
& =\int_{O(d)} \widehat{\mu}\left(U^{*}\left(\xi_{1}, \ldots, \xi_{d}\right), \xi_{d+1}\right) d \lambda(U),
\end{aligned}
$$

where $\lambda$ is the Haar probability measure on $O(d)$. Hence $v$ satisfies

$$
|\widehat{v}(\xi)|+|\widehat{\widetilde{v}}(\xi)| \lesssim|\xi|^{-\alpha / 2} \quad \forall \xi \in \mathbb{R}^{d+1},
$$

where $\widetilde{v}$ is the pushforward of $v$ under $\left(x_{1}, \ldots, x_{d}, x_{d+1}\right) \mapsto\left(x_{1}, \ldots, x_{d},-x_{d+1}\right)$. Let $\pi: \mathbb{R}^{d+1} \rightarrow \mathbb{R}$ be the map $\left(x_{1}, \ldots, x_{d}, x_{d+1}\right) \mapsto x_{d+1}$. Since spt $\mu \subseteq \mathcal{C}^{d}$, and by (6.2), the formula (6.3) can also be written as

$$
\int f d v=\int_{\mathbb{R}} \int_{S^{d-1}} f(z w, z) d \sigma(w) d \pi_{\#} \mu(z) .
$$

Hence another expression for $\widehat{v}$ is

$$
\widehat{v}(\xi)=\int e^{-2 \pi i z \xi_{d+1}} \widehat{\sigma}\left(z\left(\xi_{1}, \ldots, \xi_{d}\right)\right) d \pi_{\#} \mu(z) .
$$

Let $\omega_{d-1}$ be the surface area of $S^{d-1}$. The asymptotic formula (see [8, Appendix B])

$$
\widehat{\sigma}(\xi)=\frac{2}{\omega_{d-1}}|\xi|^{-(d-1) / 2} \cos \left(2 \pi|\xi|-\frac{\pi(d-1)}{4}\right)+O\left(|\xi|^{-(d+1) / 2}\right),
$$


gives, by taking $\xi_{d+1}=\left|\left(\xi_{1}, \ldots, \xi_{d}\right)\right|$,

$$
\begin{aligned}
& e^{\frac{i \pi(d-1)}{4}} \widehat{v}(\xi,|\xi|)+e^{\frac{-i \pi(d-1)}{4}} \widehat{v}(\xi,|\xi|) \\
& =\frac{4}{\omega_{d-1}}|\xi|^{-(d-1) / 2} \int z^{-(d-1) / 2} \cos ^{2}\left(2 \pi|\xi| z-\frac{\pi(d-1)}{4}\right) d \pi_{\#} \mu(z) \\
& \quad+O\left(|\xi|^{-(d+1) / 2}\right) .
\end{aligned}
$$

Comparing (6.4) to the above will give a contradiction, by the following identity:

$$
\lim _{r \rightarrow \infty} \int \cos ^{2}(r z+t) d \pi_{\#} \mu(z)=1 / 2 \quad \forall t \in \mathbb{R} .
$$

It remains to prove (6.6). Since $d \geq 2$ and $\alpha / 2>(d-1) / 2 \geq 1 / 2$, condition (6.1) gives $\pi_{\#} \mu \in L^{2}(\mathbb{R})$ (see e.g. [15,Theorem 3.3]). Hence $\pi_{\#} \mu \in L^{1}(\mathbb{R})$ with $\left\|\pi_{\#} \mu\right\|_{1}=1$, and (6.6) then follows by approximating $\pi_{\#} \mu$ in $L^{1}$ with a finite linear combination of characteristic functions of disjoint intervals.

\section{Further work and some questions}

The simplicity of Oberlin's argument in [17] makes it very appealing to try to adapt it to a range of different problems, such as the ones we consider here. Another problem is the following dual to the Kakeya problem, motivated by work of Wolff [21]. Suppose $E \subseteq \mathbb{R}^{d}$ contains a sphere of every radius $r \in(0,1)$. Wolff [21] proved that $E$ necessarily has Hausdorff dimension $d$. This result was proved by Kolasa and Wolff for $d \geq 3$ [12], which is much easier than the $d=2$ case. Here there is a trivial lower bound of $d-1$ for the Fourier dimension, since a single sphere in $\mathbb{R}^{d}$ has Fourier dimension $d-1$, see [15,Equation (3.42)]. For Kakeya sets there is no non-trivial bound since line segments have Fourier dimension 0 for $d \geq 2$. Despite non-trivial estimates existing for the Fourier dimension of Kakeya sets, we are unaware of any improvement over the trivial lower bound for the dual problem and pose this as a question.

Question 7.1 If $E \subseteq \mathbb{R}^{d}$ contains a sphere of every radius $r \in(0,1)$, then is it true that $\operatorname{dim}_{\mathrm{F}} E=d$ ?

For $E$ as in Question 7.1, for every $r \in(0,1)$ there is a centre $x_{r}$ such that

$$
x_{r}+r \theta \in E
$$

for all $\theta \in S^{d-1}$. One can try to adapt Oberlin's argument by defining a measure $\mu$ on $E$ as in (3.1) by, for example,

$$
\int_{E} f \mathrm{~d} \mu=\int_{0}^{1} \int_{S^{d-1}} f\left(x_{r}+r \theta\right) \phi(r) \mathrm{d} \sigma^{d-1} \theta \mathrm{d} r
$$

where $\sigma^{d-1}$ is the spherical measure on $S^{d-1}$. Using Fubini's theorem the Fourier transform of $\mu$ at $\xi \in \mathbb{R}^{d}$ is then

$$
\hat{\mu}(\xi)=\int_{S^{d-1}} \int_{0}^{1} e^{-2 \pi i\left(x_{r}+r \theta\right) \cdot \xi} \phi(r) \mathrm{d} r \mathrm{~d} \sigma^{d-1} \theta .
$$

The problem now comes that the centre $x_{r}$ depends on $r$ (not on $\theta$ for example) and so cannot be integrated out to reduce the problem to studying the Fourier transform of $\phi$. Alternatively, 
one could try to associate the Schwartz function $\phi$ with the parameter $\theta$, but this leads to a very awkward integral.

Another very intriguing problem is, of course, the Fourier analytic formulation of the Kakeya problem.

Question 7.2 If $E \subseteq \mathbb{R}^{d}$ contains a unit line segment in every direction, then is it true that $\operatorname{dim}_{\mathrm{F}} E=d$ ?

We are not aware of any improvements over $\operatorname{dim}_{\mathrm{F}} E \geq 2$ for Kakeya sets $E$. This estimate follows from Corollary 2.4. Oberlin only gives this result for $d=2$ and the improvement we obtain requires the additional geometric argument used in proving Proposition 2.2. We have some doubts about a positive answer to Question 7.2 in general.

The measures $\mu$ we use in this paper do not take into account the specific placement of the lines (or $k$-dimensional balls); indeed, when bounding the Fourier transform of $\mu$ the dependency on the translation $t_{s}$ is simply removed by making a trivial estimate. It would be interesting to try to modify the measure to account for translations, but we do not know how to do this in an effective way.

Acknowledgements We thank Pertti Mattila for helpful discussions, especially for pointing out the papers $[4,18]$. We also thank Pablo Shmerkin for helpful discussions and Tuomas Orponen for pointing out some references.

Open Access This article is licensed under a Creative Commons Attribution 4.0 International License, which permits use, sharing, adaptation, distribution and reproduction in any medium or format, as long as you give appropriate credit to the original author(s) and the source, provide a link to the Creative Commons licence, and indicate if changes were made. The images or other third party material in this article are included in the article's Creative Commons licence, unless indicated otherwise in a credit line to the material. If material is not included in the article's Creative Commons licence and your intended use is not permitted by statutory regulation or exceeds the permitted use, you will need to obtain permission directly from the copyright holder. To view a copy of this licence, visit http://creativecommons.org/licenses/by/4.0/.

\section{References}

1. Besicovitch, A.: On Kakeya's problem and a similar one. Math. Z. 27, 312-320 (1928)

2. Bourgain, J.: Besicovitch type maximal operators and applications to Fourier analysis. Geom. Func. Anal. 1, 147-187 (1991)

3. Davies, R.: Some remarks on the Kakeya problem. Math. Proc. Camb. Philos. Soc. 69, 417-421 (1971)

4. Ekström, F., Persson, T., Schmeling, J.: On the Fourier dimension and a modification. J. Fractal Geom. 2, 309-337 (2015)

5. Falconer, K.J.: Continuity properties of $k$-plane integrals and Besicovitch sets. Math. Proc. Camb. Philos. Soc. 87, 221-226 (1980)

6. Falconer, K.J.: The Geometry of Fractal Sets. Cambridge University Press, Cambridge (1985)

7. Falconer, K.J.: Fractal Geometry: Mathematical Foundations and Applications, 3rd edn. John Wiley \& Sons, Hoboken (2014)

8. Grafakos, L.: Classical Fourier analysis, Graduate Texts in Mathematics, 2nd edn., p. 249. Springer, New York (2008)

9. Hickman, J., Rogers, K. M., Zhang, R.: Improved bounds for the Kakeya maximal conjecture in higher dimensions, Amer. J. Math., (to appear), available at: arXiv:1908.05589

10. Katz, N., Tao, T.: New bounds for Kakeya problems. J. d'Anal. Math. 87, 231-263 (2002)

11. Katz, N., Zahl, J.: An improved bound on the Hausdorff dimension of Besicovitch sets in $\mathbb{R}^{3}$. J. Am. Math. Soc. 32, 2 (2017)

12. Kolasa, L., Wolff, T.: On some variants of the Kakeya problem. Pacific J. Math. 190, 111-154 (1999)

13. Marstrand, J.: Packing planes in $\mathbb{R}^{3}$. Mathematika 26, 180-183 (1979)

14. Mattila, P.: Geometry of sets and measures in Euclidean spaces, Cambridge studies in advanced mathematics, vol. 44. Cambridge University Press, Cambridge (1995) 
15. Mattila, P.: Fourier analysis and Hausdorff dimension, Cambridge Studies in Advanced Mathematics., vol. 150. Cambridge University, Cambridge (2015)

16. Mattila, P.: Hausdorff dimension, projections, intersections, and besicovitch sets. New Trends Appl. Harmonic Anal. 2, 129-157 (2019)

17. Oberlin, D.: Restricted radon transforms and unions of hyperplanes. Revista Mat. Ibero. 22, 2 (2006)

18. Oberlin, D.: Exceptional sets of projections, unions of $k$-planes and associated transforms. Israel J. Math. 202, 331-342 (2014)

19. Oberlin, R.: Two bounds for the $X$-ray transform. Math. Z. 266, 623-644 (2010)

20. Rudin, W.: Real and Complex Analysis, 3rd edn. McGraw-Hill, New York (1987)

21. Wolff, T.: A Kakeya-type problem for circles. Am. J. Math. 119, 985-1026 (1997)

Publisher's Note Springer Nature remains neutral with regard to jurisdictional claims in published maps and institutional affiliations. 